\title{
Estimación de cargas y asentamientos en pilotes debido a fricción negativa producto de licuefacción. Aplicación a terremoto del Maule 2010, Chile
}

\section{Loads and settlements estimations in piles due to negative friction caused by liquefaction.} Application to the Maule 2010 earthquake, Chile

Fecha de entrega: 11 de noviembre 2018

Fecha de aceptación: 30 de julio 2019

\section{Rodolfo Cabezas ${ }^{1}$ y Christian Ledezma ${ }^{2}$}

\begin{abstract}
${ }^{1}$ Facultad de Ingeniería y Tecnología, Universidad San Sebastián, Bellavista 7, Recoleta, Chile, rodolfo.cabezas@uss.cl
${ }^{2}$ Departamento de Ingeniería Estructural y Geotécnica, Pontificia Universidad Católica de Chile, Avda. Vicuña Mackenna 4860, 7820436, Macul, Chile, ledezma@ing.puc.cl (Orcid: 0000-0003-3821-6264)
\end{abstract}

En el contexto de un sismo de gran magnitud, el downdrag inducido por licuefacción de suelos es la sobrecarga producto del asentamiento post-licuefacción del suelo que rodea al pilote debido al reacomodo y la disminución volumétrica de las capas licuables. En muchas ocasiones, la magnitud de los asentamientos y cargas generadas por el efecto del downdrag en pilotes no está bien definida. En esta investigación se avanza en la estimación de estas cargas adicionales. Para eso se analizan casos de estudio ocurridos en el pasado terremoto del Maule de 2010, comparando metodologías de carga por fuste contra resultados de modelamiento numérico usando elementos finitos nolineales, a fin de estimar los niveles de asentamiento, los esfuerzos de corte en la interfaz suelo-pilote, la carga axial en el pilote, y la deformación volumétrica del suelo, entre otros. Como conclusión general se tiene que en los pilotes estudiados se comprueba la presencia de downdrag, el que se ve manifestado en la movilización efectiva que alcanzó el suelo en el fuste de los pilotes suroeste y noreste, de $0.2 \% y$ $0.1 \%$ respectivamente. Estas deformaciones del terreno se traducen en esfuerzos de corte adicionales sobre la interfaz entre el suelo y la estructura, con valores de cargas axiales de 45 y 36 toneladas respectivamente.

Palabras clave: downdrag, pilotes, licuefacción, sobrecarga potencial por downdrag SPD, asentamiento
During a high-magnitude earthquake, drag loads can be induced by liquefaction of soils, defined as the additional vertical load caused by the settlement after soil liquefaction around the pile due to yielding and volume decreasing in soft soils layers, causing a downdrag displacement. In several projects, the magnitude of these loads and settlements caused by downdrag in piles is not well defined. In this research, we advance towards estimating this drag loads and their impact on projects. For this purpose, a case study considering the 2010 Maule earthquake was analyzed, comparing methodologies of shaft loads against numerical modelling results using nonlinear finite element method, in order to estimate the range of settlement, shear stress at the soil-structure interface, the axial loading in the pile and the volumetric strain, among others. It can be demonstrated that the studied piles show downdrag with its consequently drag loads, which is shown as the effective mobilization in the surrounding soil of the southeast and northeast piles, with a strain of $0.2 \%$ and $0.1 \%$ respectively. These strains imply an additional shear stress in the soilstructure interface, with axial loadings of 45 and 36 ton respectively.

Keywords: downdrag, piles, liquefaction, drag load, settlement

\section{Introducción}

La licuefacción es un proceso de gran interés en geotecnia, en el que las ondas sísmicas de corte provocan un aumento en la presión del agua intersticial en un estrato de suelo sin cohesión, o con reducida cohesión (Castro, 1975), reduciendo la tensión efectiva que confina el suelo y el módulo de corte del suelo (Ishihara y Towhata, 1983), haciendo que sea más deformable progresivamente (Ferritto, 1997). Los suelos que reúnen características para presentar licuación ante presencia de un elemento iniciador, como un sismo de mayor magnitud se denominan potencialmente licuables. Dentro de las características de 
los suelos potencialmente licuables, favorece los suelos que se encuentren saturados y posean baja densidad relativa, o que sean suelos de baja plasticidad, ya que es en presencia de agua que estos tipos de suelos pueden presentar disminución en sus propiedades mecánicas de resistencia. Como este fenómeno se puede generar tanto en suelos cohesivos como en suelos no cohesivos, se acostumbra a separar el término licuefacción para describir el comportamiento de suelos sin cohesión (gravas, arenas y sedimentos de muy baja plasticidad), del término ablandamiento cíclico para describir el comportamiento de las arcillas y limos plásticos (Idriss y Boulanger, 2008).

En el caso de obras portuarias y otras obras que impliquen la presencia de pilotes en suelos saturados, este fenómeno trae consigo al menos tres efectos dañinos sobre la capacidad resistente del pilote: fricción negativa $o$ downdrag, corrimiento lateral o lateral spreading, y falla de flujo o flow failure, que actúan sobre el pilote de manera axial para el primer caso y lateralmente para los otros dos (Poulos y Davis, 1975). La Figura 1 ejemplifica la ocurrencia de downdrag, condición que provoca una carga de arrastre extra debido al descenso por el reacomodo del material granular de la o las capas que licuaron durante el sismo o por el asentamiento en el tiempo de estas capas. Si esta carga de arrastre extra es lo suficiente como para superar la capacidad de punta del pilote o la resistencia por fuste se pueden generar descensos importantes del pilote. También puede producirse sin la presencia de estas ondas y generarse por condiciones de largo plazo, incluso si el asentamiento en el suelo cercano es pequeño, se desarrollará fricción negativa en el fuste y los pilotes se someterán a carga de arrastre (Fellenius y Siegel, 2008). Hasta cierto punto a lo largo del pilote, llamado eje neutro, el asentamiento del suelo es mayor que el movimiento descendente del pilote (Matyas y Santamarina, 1994). Por debajo de este plano, el movimiento hacia abajo del pilote es mayor que el asentamiento del suelo y las tensiones de corte movilizadas actúan hacia arriba (Briaud y Tucker, 1996).

Los países con presencia de mayores sismos como Chile han experimentado este fenómeno en ocasiones, con graves daños a la superestructura, y podrían volver a ocurrir si es que no se toman las medidas de diseño suficientes (Rui et al., 2015). Debido a la sobrecarga que genera el downdrag sobre los pilotes, y que no necesariamente está bien a)
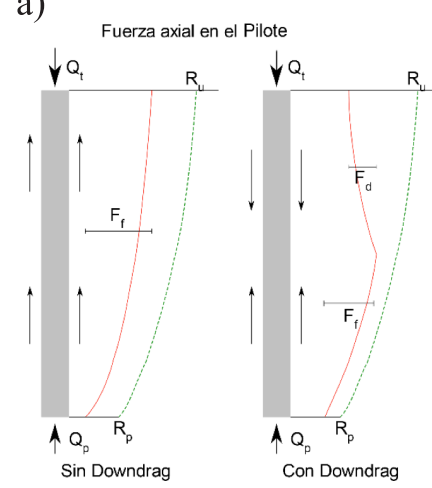

b)

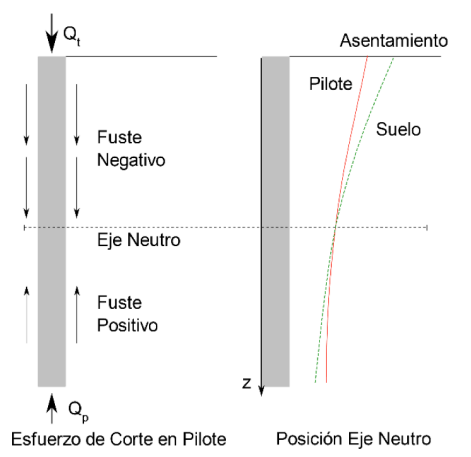

Figura 1. Distribución de fricción negativa en parte del fuste y su efecto sobre el pilote. a) Carga axial sobre el pilote sin y con downdrag o arrastramiento y b) consecuencia de sobrecarga debido a la fricción negativa mostrando la distribución de resistencia de fuste y el asentamiento total del pilote, incluyendo pilote y suelo (Briaud y Tucker, 1996).

documentada en casos prácticos, esta investigación busca contrastar una estimación de la sobrecarga y asentamiento en los pilotes que experimenten fricción negativa. En esta investigación se utilizarán casos de estudio asociados al terremoto del Maule de 2010 de posible fricción negativa por licuefacción, registrado en alguna de las cepas del puente Juan Pablo II ubicado en el río Bío-Bío, que une las ciudades de Concepción y San Pedro de la Paz con una longitud de $2.3 \mathrm{~km}$. Los datos recogidos de distintas investigaciones realizadas en el lugar post-terremoto se contrastan con los resultados del modelo numérico nolineal con el software OpenSeesPL, para luego comparar las movilizaciones de capacidad portante de los estratos y estimar las sobrecargas adicionales que habrían surgido del asentamiento post-sismo.

\section{Marco teórico}

\section{Potencial de licuefacción}

El potencial de licuefacción se define usualmente a través de la resistencia cíclica del suelo (Cyclic Resistance Ratio, CRR) (Seed et al., 1975) y la solicitación cíclica equivalente del sismo en el suelo (Cyclic Stress Ratio, CSR) (Seed e Idriss, 1971), a través de un factor de seguridad que relaciona ambos elementos. Ambas definiciones han tenido actualizaciones, tanto CRR, como la solicitación CSR, tal que valores bajo 0.9 implicarían alguna remediación al terreno (Youd e Idriss, 2001). La ecuación se define como (Idriss y Boulanger, 2008, 2010): 


$$
F S=\frac{C R R}{C S R}=\frac{\text { Cyclic Resistance Ratio }}{\text { Cylic Stress Ratio }}
$$

El parámetro CSR, que representa la solicitación a la cual se somete el terreno, queda expresado con la siguiente ecuación (Boulanger, 2014):

$$
C S R=\frac{0.65 \sigma_{v o}}{\sigma_{v o}^{\prime}} \frac{a_{\max }}{g} r_{d}
$$

donde $a_{\max }$ es la aceleración máxima del sismo, $\sigma_{\mathrm{vo}}$ es el esfuerzo vertical total, $\sigma^{\prime}{ }_{\text {vo }}$ es el esfuerzo vertical efectivo, $g$ es la aceleración de gravedad y $r_{\mathrm{d}}$ es un factor de reducción de esfuerzos según profundidad (Seed et al., 2003).

Una vez producida la licuación, y por definición, el asentamiento del suelo en el plano neutro será igual al asentamiento del pilote (Fellenius y Siegel, 2008). En el caso de un pilote con su punta apoyada sobre una capa competente que no permite el hundimiento por esta carga extra de arrastre, el único desplazamiento percibido será el acortamiento del pilote. En este caso el eje neutro tiende a situarse cerca de la parte inferior de la capa compresible (licuable), dando lugar a una gran cantidad de downdrag (Briaud y Tucker, 1996). Con esta fricción negativa $F_{N}$, la capacidad de soporte del pilote queda expresada como:

$$
Q_{T}=Q_{P}+F_{P}-F_{N}
$$

Donde $Q_{T}$ es la fuerza axial total sobre el pilote, $Q_{P}$ es la resistencia de punta, $F_{P}$ es la resistencia de fuste y $F_{N}$ es la fricción negativa debido a downdrag. Donde el fuste se calcula con los métodos convencionales de integración en contorno. Luego la máxima carga en el pilote queda expresada por:

$$
Q_{\max }=Q_{T}+F_{N}=Q_{P} F_{P}
$$

El downdrag altera el diseño convencional de pilotes, pues cuando el asentamiento vertical del suelo es mayor que el del pilote se produce la fuerza de compresión adicional (dragload) y un asentamiento excesivo (downdrag) por la fricción negativa (Kong et al., 2013). Algunos autores han estimado estas cargas como aproximadamente el 50\% de la fricción positiva del fuste antes de la licuefacción (Rollins y Hollenbaugh, 2015) o de $0.3 \mathrm{~m}$ para pilotes de $16 \mathrm{~m}$ de profundidad, correspondientes a aproximadamente un $2 \%$ de asentamiento post licuefacción (Vijayaruban et al., 2015).

\section{Asentamientos por licuefacción en pilotes}

Los asentamientos del suelo por cambios de volumen de los estratos licuables debido al drenaje de sobrepresión de poros han sido estudiados por varios investigadores y resumido recientemente (Mishra y Patra, 2018). De los estudios se concluye que la contracción de volumen después de la licuefacción depende de la densidad del estrato y de la tensión máxima de corte que el material (de preferencia arenas sueltas saturadas) ha sufrido durante la aplicación de cargas cíclicas (Ishihara y Yoshimine, 1992), o su equivalente en deformación de corte máxima. Estas conclusiones in situ fueron observadas también para arenas limpias en otros proyectos que evidenciaron licuefacción debido al sismo de 2010 (González, 2015). Otras aproximaciones relacionan los valores esperados de deformaciones volumétricas si el sismo es capaz de generar la licuación, conforme a la disipación de las presiones de poro excedentes generadas cíclicamente post-terremoto (Seed et al., 2003).

\section{Estimación de dragload debido a downdrag}

En la actualidad diversos investigadores han desarrollado modelos para estimar el desplazamiento del pilote por downdrag tanto para pilotes individuales (Rodriguez y Auvinet, 2011) como para grupos de pilotes (Kong et al., 2013). Muchas de las formulaciones corresponden a asentamientos esperados por consolidación a largo plazo, o sea en intervalos de tiempo superiores a un par de años instalados los pilotes, y no necesariamente a asentamientos de corto plazo asociados a sismos. Por tanto, el desplazamiento total posterior a la licuefacción será la superposición del asentamiento esperado de la curva de reacción del terreno a largo plazo y del adicional debido al evento sísmico (Vijayaruban et al., 2015).

Si el asentamiento adicional genera deformaciones en el terreno, o deslizamientos en la interfaz (Jeong et al., 2004), suficientes para movilizar los distintos estratos presentes en el terreno, se puede considerar entonces que los estratos licuados trabajan con fricción negativa según los métodos convencionales de capacidad de carga. Al asentarse se arrastra consigo al pilote, por lo que es intuitivo asumir que la fuerza con que el suelo arrastra al pilote (dragload) en las capas licuadas tiene una magnitud semejante y opuesta a la fricción positiva que tenían estas capas ante solicitaciones 
estáticas o previas a producirse la licuefacción.

Los asentamientos y la fricción negativa asociada también se han documentado en otros lugares, como Ciudad de México (Auvinet y Hanell, 1981), llevando incluso a guías de diseño más actualizadas (Auvinet-Guichard y Rodríguez-Rebolledo, 2017). Otros métodos para estimar potencial de licuación y métodos para estimar cargas de fuste son listados en manuales de diseño (Dirección de Vialidad, 2016), aunque no aborda una metodología de estudio ni en qué condiciones se produce este fenómeno.

\section{Caso de estudio}

Posterior al terremoto del Maule en 2010, equipos de trabajo documentaron incidencias de lateral spreading (de la Maza et al., 2015) y de downdrag inducidas por licuefacción y fallas asociadas de varios muelles de puente a través del río Bío-Bío en Concepción, siendo el puente Juan Pablo II el mejor documentado de estos casos (Vijayaruban et al., 2015). Inaugurado en 1974, atraviesa el río Bío-Bío y une las ciudades de Concepción y San Pedro de la Paz con una extensión de casi $2.3 \mathrm{~km}$. Está conformado por 2 estribos y 70 cepas (separadas $33 \mathrm{~m}$ entre sí), y sus apoyos o vanos son de $21.8 \mathrm{~m}$ de ancho (Serrano, 2014). Los pilotes fueron conformados en hormigón armado previa excavación y vaciado in situ de la mezcla, se apoyan entre los 14 y $16 \mathrm{~m}$ de profundidad y su diámetro es de $2.5 \mathrm{~m}$ en las cepas y de $1.5 \mathrm{~m}$ en los estribos. Los pilotes a su vez están sometidos a una carga esperada de $12.7 \mathrm{MN}$, la que se reparte $5.9 \mathrm{MN}$ por fricción y $6.8 \mathrm{MN}$ por capacidad de punta, equivalentes a $1.4 \mathrm{MPa}$ en la superficie de contacto (Vijayaruban et al., 2015).

De los análisis se evidenció que el lado norte del puente (Concepción) fue el que resultó con mayores daños de asentamiento además de sufrir el desplazamiento lateral (Yen et al., 2011). De la Figura 2 se puede observar que las cepas No. 45 y No. 60 presentan asentamientos del orden 90 y $80 \mathrm{~cm}$ aproximado respectivamente, además de que el eje longitudinal izquierdo de aguas arriba desarrolló mayores asentamientos que el eje longitudinal derecho de aguas abajo (Serrano, 2014).

Para identificar qué pilotes quedan embebidos en estratos potencialmente licuables, la Figura 3 muestra el perfil esperado donde se emplazan los pilotes (Vijayaruban et a)

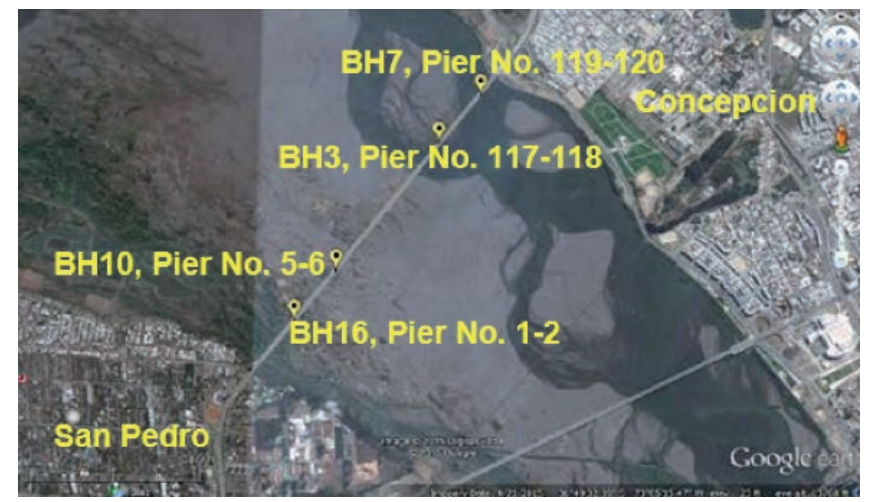

b)

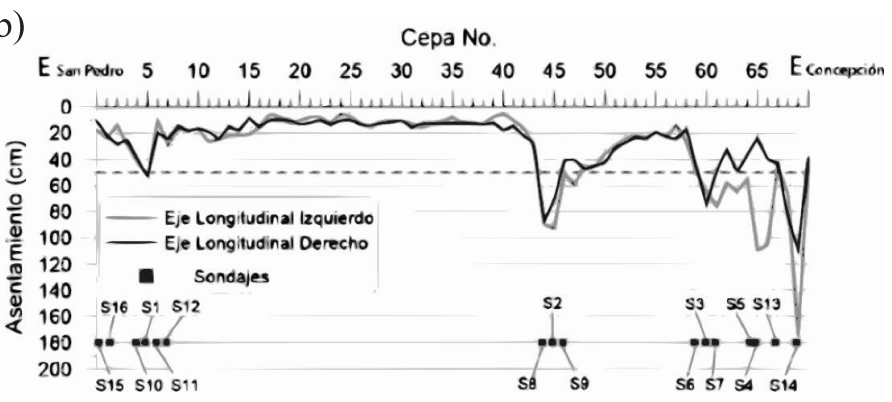

Figura 2: Asentamientos post sismo del puente Juan Pablo II. a) Ubicación de sondajes BH con los pilotes de fundación respectivos (Vijayaruban et al., 2015) y b) registro de asentamientos (Serrano, 2014)

a)

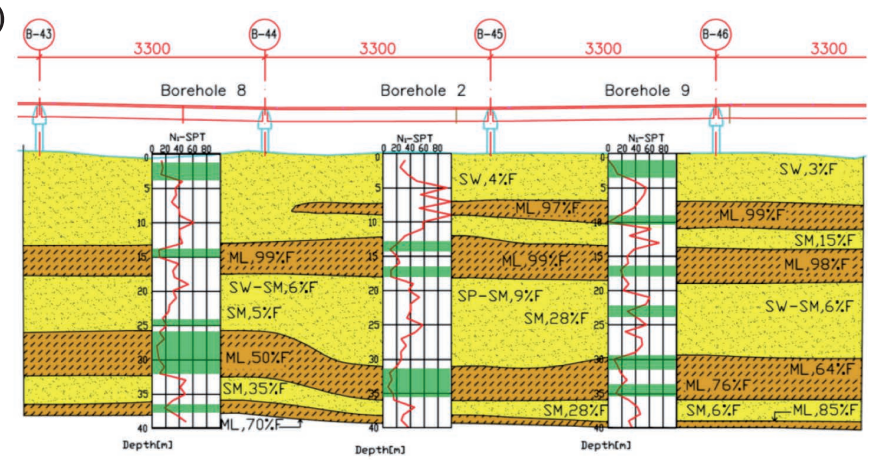

b)

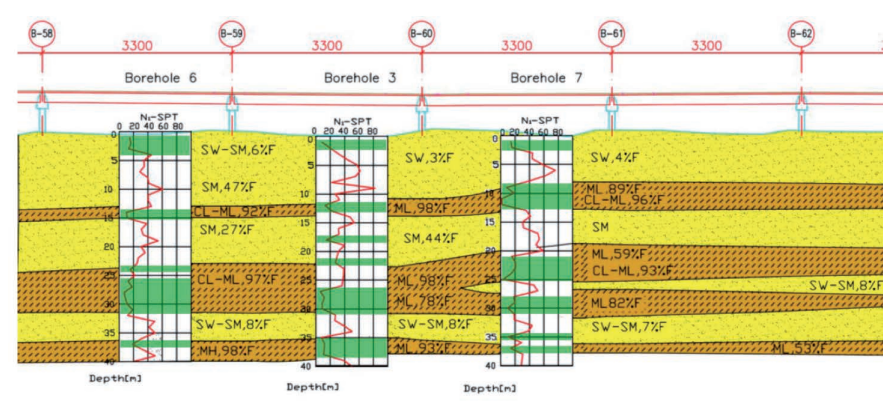

Figura 3: Perfil estratigráfico con estratos posiblemente licuables, marcadas con color verde (González, 2015). a) Sondaje 2 y b) Sondaje 7 
al., 2015), donde para valores de SPT $\left(\mathrm{N}_{1}\right)_{60}$ por debajo de 30 golpes/pie es posible considerar zonas potenciales a ser licuables (González, 2015). Las muestras fueron obtenidas de sondajes y las propiedades de los suelos fueron obtenidas de laboratorio, tal como se resume en la Tabla 1. Los resultados de los ensayos disponibles indican una fuerte variabilidad del SPT después de la corrección de datos (González, 2015), es decir, existe una importante estratificación entre suelos sueltos y densos y lentes de finos.

Tabla 1: Caracterización de tipos de suelo en el puente Juan Pablo II, usados para el modelamiento (González, 2015)

\begin{tabular}{|c|c|c|c|c|}
\hline Suelo & $\begin{array}{c}\text { Cohesión } \\
c, \\
\mathrm{kPa}\end{array}$ & $\begin{array}{c}\text { Ángulo de } \\
\text { fricción } \\
\text { máximo } \phi \\
\text { grados }\end{array}$ & $\begin{array}{c}\text { Peso unitario } \\
\text { saturado } \gamma_{\text {sat }}, \\
\mathrm{kN} \mathrm{m}^{3}\end{array}$ & $\begin{array}{c}\text { Coeficiente de } \\
\text { empuje en reposo } \\
K_{0}\end{array}$ \\
\hline $\begin{array}{c}\text { Limos } \\
\text { blandos }\end{array}$ & 14.7 & 25 & 18.6 & 0.58 \\
\hline $\begin{array}{c}\text { Limos } \\
\text { compactos }\end{array}$ & 14.7 & 28 & 18.6 & 0.53 \\
\hline $\begin{array}{c}\text { Arena } \\
\text { limosa } \\
\text { media }\end{array}$ & 4.9 & 37 & 18.6 & 0.40 \\
\hline $\begin{array}{c}\text { Arena } \\
\text { limosa densa }\end{array}$ & 4.9 & 39 & 19.6 & 0.37 \\
\hline
\end{tabular}

\section{Perfil del suelo}

Es posible definir un perfil estratigráfico simplificado, representativo del suelo de fundación en el cual fueron colocados los pilotes. En la Figura 4 se muestran los perfiles del suelo correspondientes a los sondajes 2 y 7 , ubicados en el suroeste y noreste del puente, respectivamente.

Donde la resistencia por fuste $f$ desarrollada en el contorno del pilote se considera constante a partir de la profundidad de 15 veces el diámetro (Das, 2012). En nuestro caso en que los pilotes cuentan con una profundidad de $16 \mathrm{~m}$, el parámetro $f$ aumenta linealmente de manera que se puede calcular como $f_{\text {prom }}$ para pilotes hincados de bajo desplazamiento y en correlación con resultados del ensayo de penetración estándar (Das, 2012) según la siguiente ecuación:

$$
f_{\text {prom }}=0.01 p_{a}\left(\bar{N}_{60}\right)
$$

Donde $\left(\bar{N}_{60}\right)$ es el valor promedio por estrato de la resistencia a la penetración estándar y $p_{a}$ es la presión atmosférica $\left(\approx 100 \mathrm{kN} / \mathrm{m}^{2}\right)$.
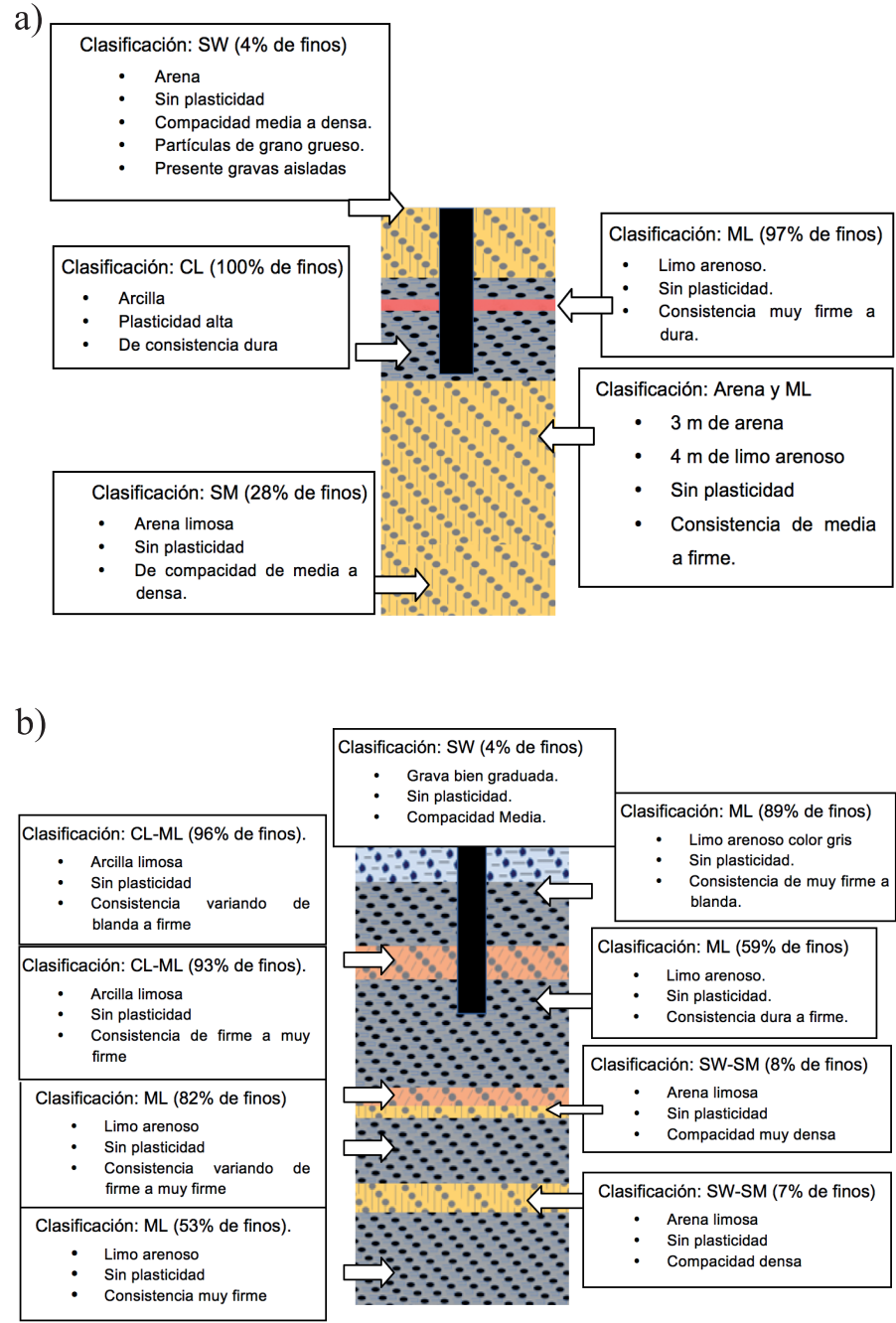

c)

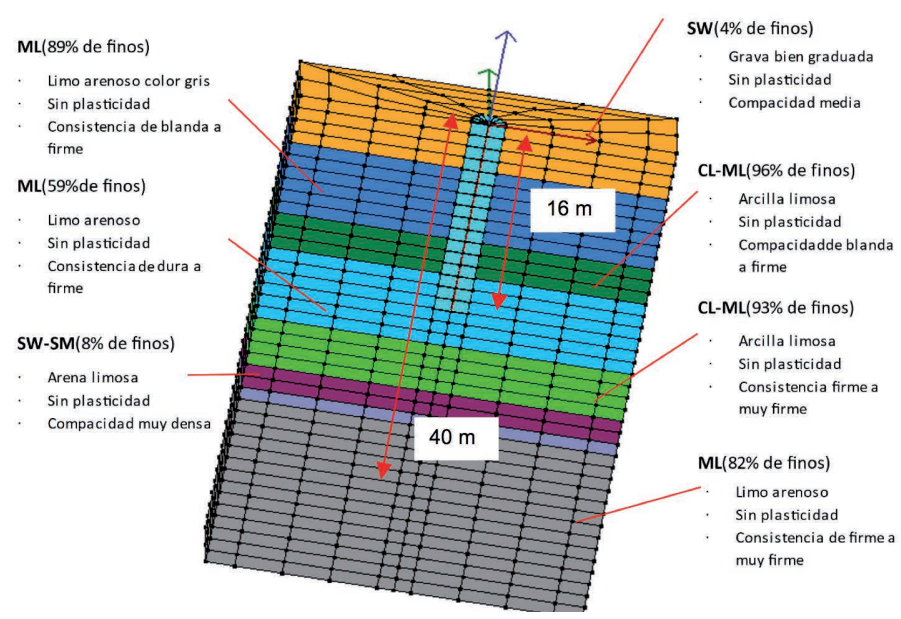

Figura 4: Perfiles estratigráficos y representación gráfica en modelo OpenSees, a) pilote emplazado en Sondaje 2, b) pilote emplazado en Sondaje 7 y c) representación gráfica de pilote en S7 en software OpenSees 
Con las ecuaciones para estimar la fricción negativa y los perfiles de suelo identificados se puede obtener la máxima carga teórica por fricción negativa, considerando todos los estratos que interactúan con los pilotes de los sondajes 2 y 7. Con lo anterior, la carga por fricción negativa $Q$ para los pilotes S2 y S7 sería aproximadamente de $Q_{-s 2}=Q_{\text {arena }}+$ $Q_{\text {fino }}=843 \mathrm{kN} \mathrm{y} Q_{-s 7}=Q_{\text {arena }}+Q_{\text {fino }}=1785 \mathrm{kN}$.

\section{Estimación de asentamiento mediante software OpenSeesPL}

OpenSees es un software de elementos finitos no lineales de integración explícita, por lo que permite recrear el comportamiento del suelo con cálculos dinámicos directamente con el registro de aceleraciones (www. opensees.berkeley.edu). Cuenta con distintos modelos constitutivos focalizados en arena o finos y distintas publicaciones de validación con mediciones históricas.

Para el análisis se usaron las aceleraciones registradas en la estación de Concepción. Este acelerómetro registró una aceleración horizontal máxima de $-0.402 \mathrm{~g}$ para la componente longitudinal y de $0.29 \mathrm{~g}$ para la componente transversal. La Figura 5 muestra la comparación de los gráficos de aceleraciones registrados en superficie por el acelerógrafo en cuestión y los registros calibrados por el programa a nivel de superficie y a nivel del sello de los pilotes, con el objetivo de asegurar que el input de aceleraciones en la base del pilote sea representativo de lo que se registró.

\section{Estimación de asentamiento mediante software OpenSeesPL}

El modelo constitutivo utilizado en el modelo es el propuesto para arenas (Yang et al., 2003). En primera instancia, el modelo define la curva de tensión-deformación en términos del comportamiento del esfuerzo de corte $\tau$, como:

$$
\tau=G \frac{\gamma}{1+\gamma / \gamma_{s_{\max }}}
$$

donde $G$ es el módulo de corte elástico, $\gamma$ es la deformación de corte y $\gamma_{\text {smax }}$ es la deformación de corte máxima o residual, para grandes deformaciones.

Para eso se define una superficie de fluencia del material
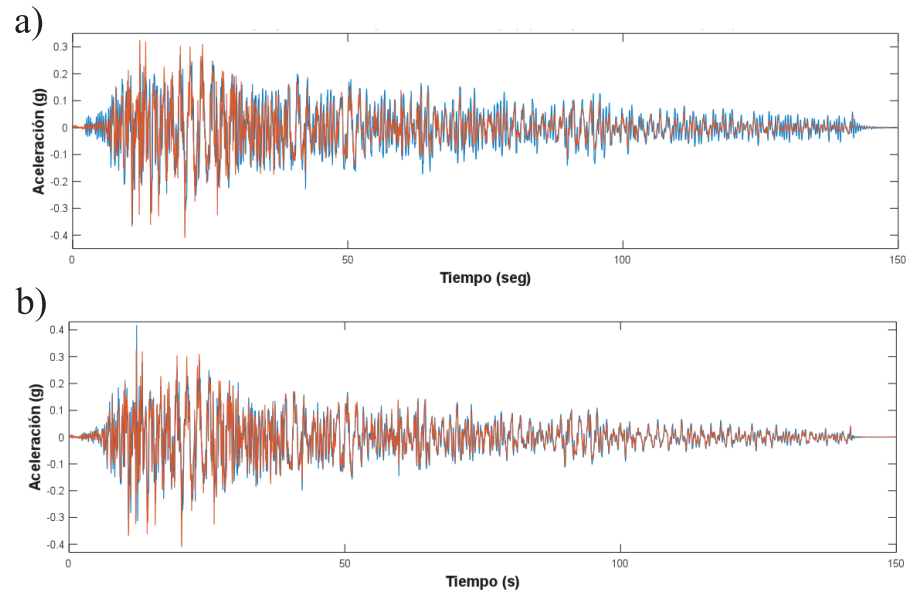

c)

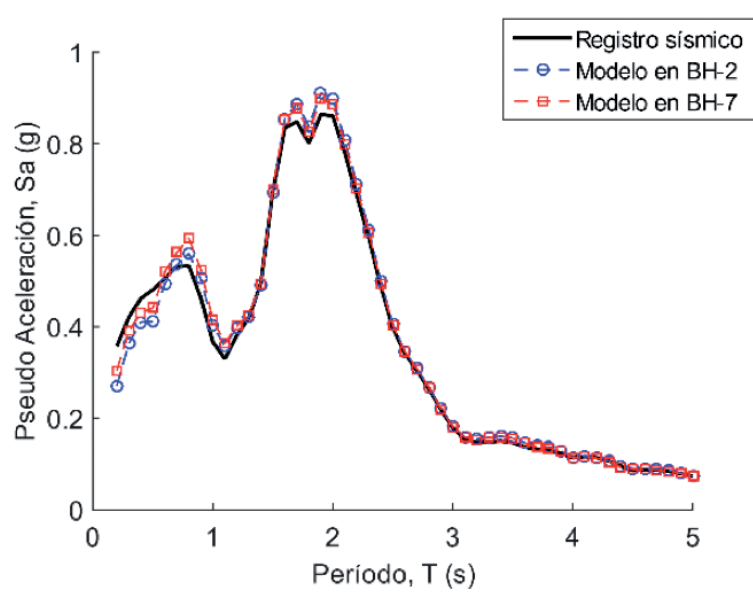

Figura 5: Superposición de registros RENADIC versus OpenSeesPL. a) S2 en superficie, b) S7 en superficie y c) espectro de respuesta, evaluado en la base del pilote a $16 \mathrm{~m}$ de profundidad

como una variante de los modelos CAP, tal que:

$$
F=\frac{3}{2}\left(\boldsymbol{s}-p^{\prime} \boldsymbol{\alpha}\right):\left(\boldsymbol{s}-p^{\prime} \boldsymbol{\alpha}\right)-M^{2}{p^{\prime}}^{2}=0
$$

donde $\boldsymbol{s}$ es el tensor desviador, $p$ ' es la tensión media efectiva, $\alpha$ es un tensor desviador de segundo orden, evaluado en la tensión media $p^{\prime}, M$ define el tamaño de la superficie de fluencia $\mathrm{y}:$ denota un producto tensorial.

Una vez alcanzada la superficie de fluencia, el material se moviliza a través de un potencial de flujo $P$ en función de la transformación de fase (PT), que indica cuando el material se vuelve licuable. Luego $P$ que será función dependiendo si el comportamiento es contractante o dilatante, tal que:

$$
P^{\prime}=\left(1-\frac{\eta}{\eta_{p t}}\right) d_{1}\left(\gamma_{d}\right)^{d_{2}} ; P^{\prime}=\left(1-\operatorname{sign}(\dot{\eta}) \frac{\eta}{\eta_{p t}}\right)\left(c_{1}+c_{2} \gamma_{c}\right)
$$


Donde $\eta$ es la razón de esfuerzos, $\eta=\left(\sqrt{\frac{3}{2} s: s}\right) / p^{\prime}, d_{1}$ y $d_{2}$ son parámetros de ajuste del modelo para comportamiento dilatante, $c_{1}$ y $c_{2}$ y son parámetros de ajuste del modelo para comportamiento contractante, $\gamma_{d}$ y $\gamma_{c}$ son escalares relacionados con la deformación.

Este modelo define la contracción y la licuefacción con 3 parámetros cada uno. El parámetro $c_{1}$ dicta la tasa de contracción (o aumento del exceso de la presión de poros); los parámetros de dilatancia, $d_{1}$ y $d_{2}$, influyen sólo cuando la carga de corte está por fuera de la superficie de transformación de fase (Elgamal et al., 2003). Los otros tres parámetros de licuefacción llamados $l_{1}, l_{2}, \mathrm{y} l_{3}$, están relacionados con la movilidad cíclica 0 , como los define el manual de usuario del programa: parámetros que controlan el mecanismo de acumulación de deformación por corte perfectamente plástico inducido por licuefacción ( $\mathrm{Lu}$ et al., 2011).

Finalmente, la regla de endurecimiento del material se define a través de un parámetro $\mu$, tal que:

$$
\mu=\frac{M_{m+1}}{M_{m}}\left(s-p^{\prime} \boldsymbol{\alpha}_{m}\right)-\left(s-p^{\prime} \boldsymbol{\alpha}_{m+1}\right)
$$

Donde $M_{m}$ y $M_{m+1}$ es el módulo de endurecimiento del material para el estados de carga $m$ y $m+1$ respectivamente.

Para ejemplificar el modelo, el dominio de soluciones para un esfuerzo medio y la movilización de la carga durante la carga cíclica se presentan en las Figuras 6a) y 6b), respectivamente. a)

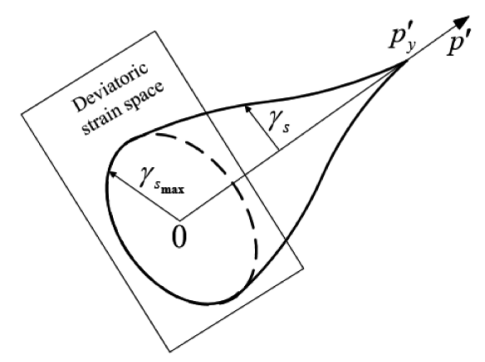

b)

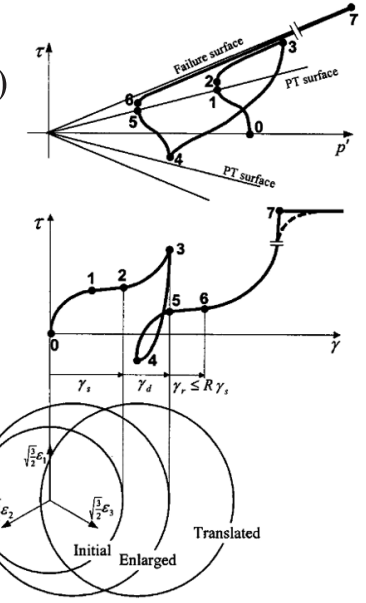

Figura 6: Elementos base de modelo constitutivo en OpenSeesPL (Kramer y Elgamal, 2001). a) Dominio bajo niveles de confinamiento efectivo y b) tensión octaédrica $\tau$ versus confinamiento efectivo, deformación por corte y fluencia
Para efectos del caso de estudio, la Tabla 2 muestra los valores recomendados para estos parámetros y que fueron usados en la modelación de S2 y S7.

Tabla 2: Valores usados para parámetros de dilatancia y licuefacción en el modelo

\begin{tabular}{|c|c|c|c|c|}
\hline $\begin{array}{c}\text { Pará- } \\
\text { metro }\end{array}$ & $\begin{array}{c}\text { Arena suelta } \\
(\mathrm{DR}=15-35 \%)\end{array}$ & $\begin{array}{c}\text { Arena media } \\
(\mathrm{DR}=35-65 \%)\end{array}$ & $\begin{array}{c}\text { Arena media } \\
\text { densa } \\
(\mathrm{DR}=65-85 \%)\end{array}$ & $\begin{array}{c}\text { Arena densa } \\
(\mathrm{DR}=85 \%-100 \%)\end{array}$ \\
\hline$c_{1}$ & 0.21 & 0.07 & 0.05 & 0.03 \\
\hline$d_{1}$ & 0.0 & 0.4 & 0.6 & 0.8 \\
\hline$d_{2}$ & 0 & 2 & 3 & 5 \\
\hline$l_{1}, \mathrm{kPa}$ & 10 & 10 & 5 & 0 \\
\hline$l_{2}$ & 0.02 & 0.01 & 0.003 & 0.000 \\
\hline$l_{3}$ & 1 & 1 & 1 & 0 \\
\hline
\end{tabular}

DR: densidad relativa

\section{Resultados}

Los resultados obtenidos de los análisis dinámicos realizados en el software para los dos pilotes en estudio se presentan a continuación, en las Figuras 7 y 8 para los pilotes S2 y S7 respectivamente. Para esto se consideró que los pilotes trabajaban de manera aislada debido a la separación en el eje del puente y que se da tiempo suficiente después del sismo para asegurar la disipación de la sobrepresión de poros. Para cada caso de estudio se recogen los resultados de asentamiento vertical del pilote, deformación unitaria vertical en el suelo, esfuerzo de corte en el suelo, y esfuerzo normal en el suelo.

Para el pilote 2 se obtiene que el asentamiento estimado por el software es de $100 \mathrm{~cm}$ aproximadamente. Además, se observa un esfuerzo de corte en el terreno que a una profundidad de la punta del pilote es de aproximadamente $7.5 \mathrm{kPa}$, y a la profundidad de $23.1 \mathrm{~m}$ el esfuerzo de corte llega al máximo con un valor $17.4 \mathrm{kPa}$. En la Figura 7c), la deformación del terreno es del orden de $0.2 \%$, valor que se mantiene casi constante en la longitud del pilote pero que se ve significativamente aumentado en los estratos siguientes a la punta del pilote llegando a una deformación máxima de $5.1 \%$ aproximadamente a $\operatorname{los} 23.1 \mathrm{~m}$ de profundidad. El aumento de esta deformación coincide también con el aumento del esfuerzo de corte y el normal, valores que descienden hasta cero llegando a los $40 \mathrm{~m}$ de profundidad.

Para el Pilote S2 se obtiene que el asentamiento estimado es de aproximadamente $41 \mathrm{~cm}$. El esfuerzo de corte registrado en la punta del pilote es de casi $7 \mathrm{kPa}$, mientras 
a)

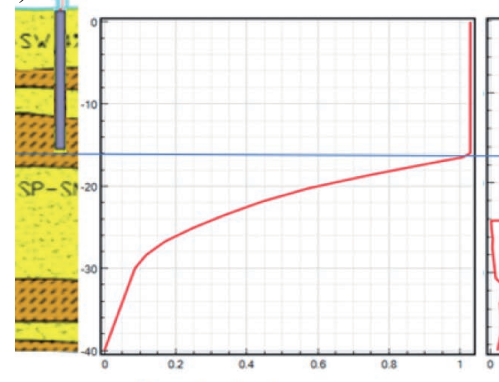

c)

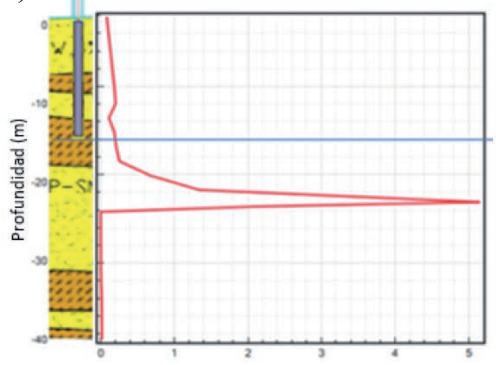

Deformación vertical, \% b)

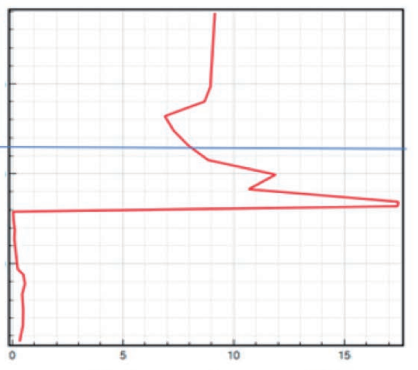

d)

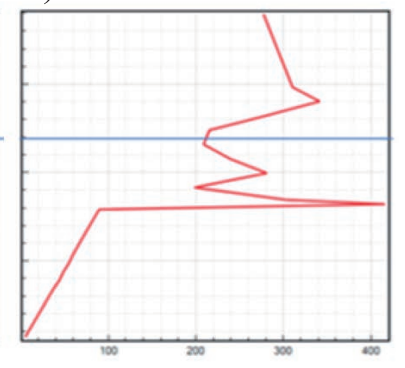

Esfuerzo normal, $\mathrm{kPa}$

Figura 7: Resultados para pilote S2. a) Asentamiento en nodos a lo largo del eje del pilote, b) esfuerzo de corte, c) deformación vertical, d) esfuerzo normal

a)

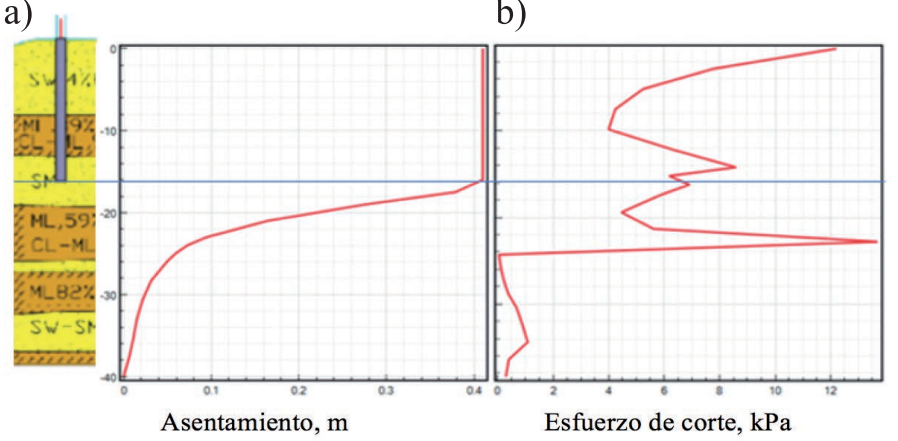

c)

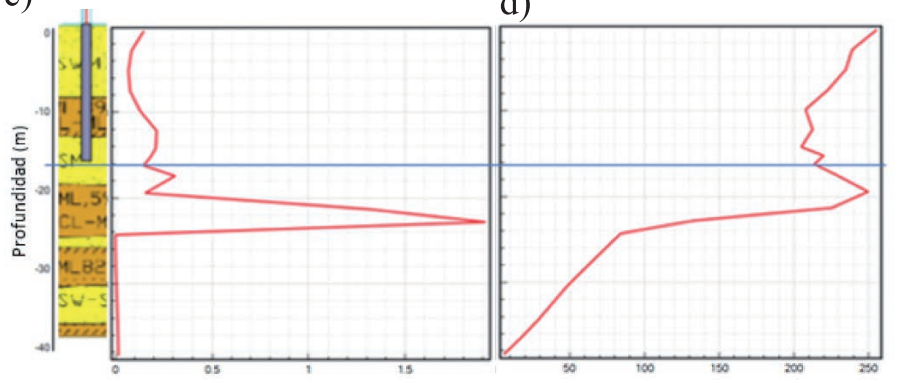

c) Deformación vertical, \%

d) Esfuerzo normal, $\mathrm{kPa}$

Figura 8: Resultados para pilote S7. a) Asentamiento en nodos a lo largo del eje del pilote, b) esfuerzo de corte, c) deformación vertical, d) esfuerzo normal

que el máximo registro se da en la profundidad de 22.8 $\mathrm{m}$ con un valor de $13.7 \mathrm{kPa}$ aproximadamente. En el caso del S7, la deformación vertical de los estratos es de aproximadamente $0.1 \%$ en los primeros metros del pilote, valor que aumenta en los metros finales del pilote llegando a una deformación de $0.2 \%$ aproximadamente en la punta del pilote, y que como hemos visto, este es un valor suficiente para generar una movilización en el terreno y por ende un arrastre en el pilote. A diferencia del S2 en donde la deformación volumétrica máxima alcanzó 5\%, el S7 solo llegó al 1.9\% de deformación aproximadamente.

Comparativamente entre los gráficos de esfuerzo de corte de S2 y S7 se observa que para los pilotes del S2 el esfuerzo de corte tiene valores casi constantes a lo largo de la longitud del pilote. En cambio, para los pilotes del S7 la respuesta máxima en la punta del pilote tiene un valor cercano a los $8 \mathrm{kPa}$, valor que decrece hasta los $10 \mathrm{~m}$ de profundidad a un valor de $4 \mathrm{kPa}$.

La Figura 9 muestra la distribución de esfuerzos de corte en la longitud de los pilotes. De la integración directa de estos esfuerzos se puede llegar a un valor estimativo del esfuerzo de corte o downdrag ejercido por el terreno movilizado por el asentamiento debido a la licuefacción, estimado aproximadamente en 45 y 36 ton para los pilotes S2 y 57 respectivamente. a)

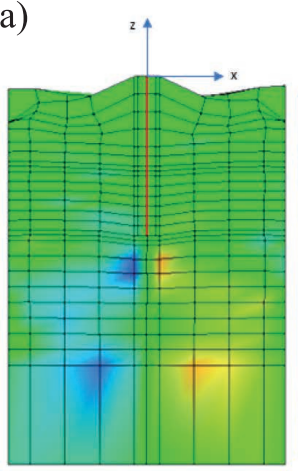

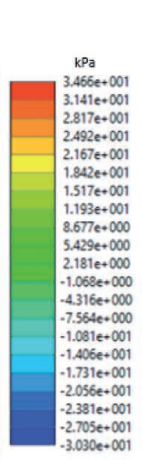

b)

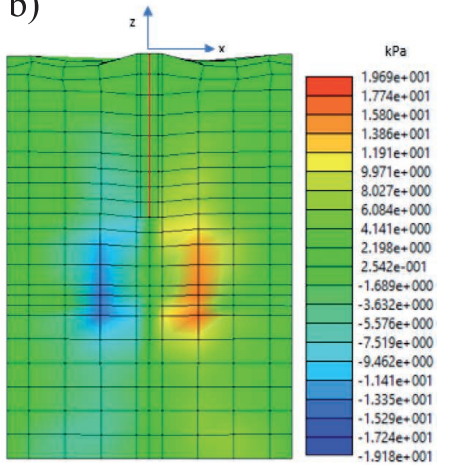

Figura 9: Distribución de esfuerzo de corte en el entorno de los pilotes después del sismo. a) Sondaje 2 y b) Sondaje 7

\section{Análisis de sobrecarga por downdrag}

Para la estimación de sobrecarga potencial por downdrag (SPD), generada en los pilotes ubicados en el S2, presenta una menor magnitud en comparación a los pilotes ubicados en el S7. Este dato no es menor considerando que el asentamiento fue mayor en los pilotes cercanos al sondaje 2. En dichos casos, la estimación de sobrecarga potencial producto del downdrag corresponde al $18 \%$ del fuste total inicial. Para los pilotes ubicados en el sondaje 7 la estimación de sobrecarga potencial producto del downdrag corresponde al $25 \%$ del fuste total inicial. 
A pesar de que el sondaje 7 presenta un 7\% mayor de SPD, lo que se observó en la realidad es que los pilotes cercanos a este sondaje presentaron un asentamiento menor a los del sondaje 2. Una explicación a esta observación es que los estratos licuables a profundidades mayores de $16 \mathrm{~m}$ tuvieron un mayor efecto de movilización en los estratos superiores lo que provocó un mayor asentamiento.

Otro factor que podría influenciar en que los pilotes del S7 presenten una mayor sobrecarga potencial por downdrag, pero menor asentamiento, es que el suelo observado bajo la punta del pilote es del tipo limo arenoso de consistencia dura a firme con altos valores de SPT. En comparación a lo que existe bajo la punta de los pilotes en el S2 en donde el suelo cuenta con un estrato de arena limosa de compacidad media al que le sigue un estrato de arcilla débilmente limosa, también de compacidad fina y ambos estratos con bajos valores de SPT, lo que supondría estratos no compactos.

Del análisis de sobrecargas se ve una diferencia notoria entre los valores obtenidos de métodos empíricos, inicialmente estimado en $6 \mathrm{MN}$ (Vijayaruban et al., 2015) contra los obtenidos por el modelo. Según esto último, debido a la movilización negativa de los estratos y la fricción asociada, se obtienen valores de $0.5 \mathrm{MN}$ y $0.4 \mathrm{MN}$ para los pilotes S2 y S7, respectivamente. Por lo anterior, se estima que el asentamiento es de $30 \mathrm{~cm}$ aproximadamente registrado bajo los pilotes sujetos a licuación superior es mucho mayor a los $22 \mathrm{~mm}$ que generarían sólo por concepto de sobrecarga efectiva. Se puede concluir entonces que hay un asentamiento general, y consecuentemente el pilote baja, pero no todos los estratos que interactúan con el pilote aportan una alta componente de sobrecarga por downdrag.

\section{Asentamiento por downdrag}

Los asentamientos totales obtenidos por el modelo para S2 y S7 fueron de $100 \mathrm{~cm}$ y $41 \mathrm{~cm}$ respectivamente. Estos resultados son comparables a los asentamientos registrados in-situ post-terremoto, donde los pilotes más solicitados S2 y S7 se asentaron aproximadamente $100 \mathrm{~cm}$ y $60 \mathrm{~cm}$ respectivamente. Si se compara con la estimación de asentamientos esperados, se comprueba que la mayor parte del material licuado se da a mayor profundidad, bajo el sello del pilote, como se notó en las primeras campañas de reconocimiento posteriores al terremoto (Bray y Frost, 2010).
De los gráficos se observa que los pilotes tienen una deformación vertical suficiente para movilizar el terreno y producir downdrag, que se traduce en el arrastre de los pilotes hacia abajo. De estas movilizaciones, la generada en S2 tiene una mayor magnitud en comparación con S7, superior en un $0.1 \%$. Esto concuerda con el asentamiento generado en S2, que es $60 \mathrm{~cm}$ mayor al de S7.

Que la deformación sea mayor en S2 puede deberse en que también presenta un mayor esfuerzo de corte y esfuerzo normal en el pilote. Además, otro motivo por el que los pilotes de S2 presenten un mayor asentamiento es por los estratos presentes en la longitud del pilote y posterior a la punta de este, donde existen arenas y estratos de densidad media.

\section{Conclusiones}

Es necesario considerar los efectos de downdrag por licuefacción para proyectos con fundaciones profundas desarrollados en países sísmicos como Chile. Aunque aún no es posible identificar la magnitud con la que actuará este fenómeno, con las herramientas disponibles en la actualidad sí se puede lograr un estimado razonable para así evaluar mejor los factores de seguridad en los diseños de las fundaciones.

De la aplicación del modelo y la calibración contra los datos registrados posterior al terremoto de 2010, a pesar de que solo un modelo (S2) alcanzó aproximadamente el asentamiento registrado en terreno de casi un metro, con el asentamiento reproducido en $\mathrm{S} 7 \mathrm{de} 40 \mathrm{~cm}$ versus $10 \mathrm{~cm}$ aproximados registrado in-situ es suficiente para confirmar el efecto de downdrag en los pilotes. El asentamiento mayor (S2) se presentó en estratos de densidad media a baja con espesores mayores comparativamente con los estratos de S7. Esto produjo un mayor reacomodo del material, resultando en una disminución volumétrica mayor y por lo tanto un esfuerzo de corte mayor en el fuste de esos pilotes. El método utilizado para calcular la sobrecarga potencial por downdrag (SPD) entendido como la movilización completa del estrato, debe ser revisado, ya que su metodología se basa en los valores de ensayos SPT para suelos que no licúan. Como se utilizó para obtener la resistencia de fuste en sentido descendente (fricción negativa), asumiendo un comportamiento ideal, se provoca que para estratos más densos o competentes se puede sobreestimar el valor de sobrecarga potencial por downdrag. Esto se observó en 
S7, en donde su valor de SPD total (equivalentes a 1700 $\mathrm{kN}$ aproximados) fue mucho mayor al de $\mathrm{S} 2$ (equivalentes a $850 \mathrm{kN}$ aproximados) porque sus estratos presentaban mayores índices de SPT. Valores muy distintos se obtuvieron del modelo, donde el downdrag estimado para $\mathrm{S} 2$ fue de 45 ton (equivalentes a $500 \mathrm{kN}$ aproximados) corresponde a un $53 \%$ de la sobrecarga potencial por downdrag (SPD) calculado en la sección de resultados. El downdrag estimado para S7 de 36 ton (equivalentes a 400 $\mathrm{kN}$ aproximados) corresponde a un $22.5 \%$ de la sobrecarga potencial por downdrag (SPD).

Los efectos debidos a la licuefacción deben ser abordados con más detalle en nuestros códigos y manuales de diseño, ya que como se observó en este trabajo, la licuefacción es un fenómeno que trae otros efectos dañinos sobre el terreno y las construcciones. Se debe trabajar en buscar métodos de compactación en suelos licuables cercanos al contorno del pilote para así disminuir la probabilidad de que exista downdrag.

\section{Referencias}

Auvinet, G. and Hanell, J.J. (1981). Negative skin friction on piles in Mexico City clay. Proceedings $10^{\text {th }}$ International Conference on Soil Mechanics and Foundation Engineering, Stockholm, Sweden, vol. 2, 599-604

Auvinet-Guichard, G. and Rodríguez-Rebolledo, J. (2017). Criteria for the design of friction piles subjected to negative skin friction and transient loads. Ingeniería, Investigación y Tecnología 18(3), 279-292

Bray, J., and Frost, J. (2010). Geo-Engineering reconnaissance of the 2010 Maule, Chile Earthquake. NSF-GEER Association Team. California, USA

Briaud, J. and Tucker, L. (1996). Design and construction guidelines for downdrag on uncoated and bitumen-coated piles. National Cooperative Highway Research Program Transportation Research Board, Washington, DC, USA

Castro, G. (1975). Liquefaction and cyclic mobility of saturated sands. Journal of Geotechnical Engineering Division 101(6), $551-569$

Das, B.M. (2012). Fundamentos de ingeniería de cimentaciones. Séptima edición, Cengage Learning Editores, México de la Maza, G., Williams, N., Sáez, E., Rollins, K. y Ledezma, C. (2015). Lateral spreading inducido por licuación en Lo Rojas, Coronel, estudio de terreno y modelo numérico. Obras y Proyectos 17, 106-115

Dirección de Vialidad. (2016). Manual de Carreteras Volumen Nº3: Instrucciones y Criterios de Diseño. Ministerio de Obras Públicas, Santiago, Chile

Elgamal, A., Yang, Z., Parra, E. and Ragheb, A. (2003). Modeling of cyclic mobility in saturated cohesionless soils. International Journal of Plasticity 19(6), 883-905

Fellenius, B. and Siegel, T. (2008). Pile drag load and downdrag in a liquefaction event. Journal of Geotechnical and Geoenvironmental Engineering 134(9), 1412-1416

Ferritto, J.M. (1997). Seismic design criteria for soil liquefaction. Report NFESC-TR-2077-SHR Naval Facilities Engineering Service Center. California, USA

González, V.P. (2015). Puentes afectados por licuefacción del terreno de fundación debido al terremoto 27F. Tesis de Magíster, Universidad de Chile

Idriss, I. and Boulanger, R. (2008). Soil liquefaction during earthquakes. Earthquake Engineering Research Institute EERI. California, USA

Idriss, I. and Boulanger, R. (2010). CPT and SPT based liquefaction triggering procedures. Department of Civil and Environmental Engineering. University of California, USA

Ishihara, K. and Towhata, I. (1983). Sand response to cyclic rotation of principal stress directions as induced by wave loads. Soils and Foundations 23(4), 11-26

Ishihara, K. and Yoshimine, M. (1992). Evaluation of settlements in sand deposits following liquefaction during earthquakes. Soils and Foundations 32(1), 173-188

Jeong, S., Lee, K. and Lee, C. (2004). Slip-effect at the pile-soil interface on dragload. Computers and Geotechnics 31(2), 115126

Kong, G., Liu, H., Yang, Q., Liang, R. Y. and Zhou, H. (2013). Mathematical model and analysis of negative skin friction of pile group in consolidating soil. Mathematical Problems in Engineering, doi:10.1155/2013/956076 
Kramer, S. and Elgamal, A. (2001). Modeling soil liquefaction hazards for performance-based earthquakes engineering. Pacific Earthquake Engineering Research Center PEER, Berkeley, USA

Lu, J., Elgamal, A. and Yang, Z. (2011). OpenSeesPL: 3D Lateral pile-ground interaction user manual (Beta 1.0). http:// www.soilquake.net/openseespl/OpenSeesPL_UserManual.pdf

Matyas, E. and Santamarina, J. (1994). Negative skin friction and the neutral plane. Canadian Geotechnical Journal 31(4), $591-597$

Mishra, A. and Patra, N. (2018). Dragload of pile groups in consolidating non-Darcian clays. Proceedings of the Institution of Civil Engineers - Geotechnical Engineering 171(4), 295-309

Poulos, H. and Davis, E. (1975). Prediction of downdrag forces on end-bearing piles. Journal of Geotechnical Engineering 101(2), 189-204

Rodriguez, J. y Auvinet, G. (2011). Modelado numérico del comportamiento de un pilote sometido a fricción negativa y cargas accidentales. Pan-American Canadian Geotechnical Conference. Ontario, Canada

Rollins, K. and Hollenbaugh, J. (2015). Liquefaction induced negative skin friction from blast-induced liquefaction tests with auger-cast piles. $6^{\text {th }}$ International Conference on Earthquake Geotechnical Engineering. Christchurch, New Zealand

Rui, W., Lui, X. and Zhang, J.-M. (2015). Analysis of seismic pile response on liquefiable ground using a constitutive model for large post-liquefaction deformation. $\sigma^{\text {th }}$ International Conference on Earthquake Geotechnical Engineering, Christchurch, New Zealand

Seed, H.B. and Idriss, I.M. (1971). Simplified procedure for evaluating soil liquefaction potential. Journal of the Soil Mechanics and Foundations Division 97(9), 1249-1273
Seed, H.B., Maksidi, F.I., Idriss, I.M. and Lee, K.L. (1975). The slides in the San Fernando Dams during the earthquake of February 9, 1971. Journal of the Geotechnical Engineering Division 101(7), 651-688

Seed, R.B., Cetin, K.O., Moss, R.E., Kammerer, A.M., Wu, J., Pestana, J.M., Riemer, M.F., Sancio, R.B., Bray, J.D., Kayen, R.E. and Faris, A. (2003). Recent advances in soil liquefaction engineering: a unified and consistent framework. In Proceedings of the $26^{\text {th }}$ Annual ASCE Los Angeles Geotechnical Spring Seminar, Long Beach, CA, USA

Serrano, C.B. (2014). Efectos de la licuefacción en puentes fundados en pilotes. Tesis de magíster, Pontificia Universidad Católica de Chile, Santiago, Chile

Vijayaruban, V., Muhunthan, B. and Fellenius, B. (2015). Liquefaction-induced downdrag on piles and drilled shafts. $6^{\text {th }}$ International Conference on Earthquake Engineering. Christchurch, New Zealand

Yang, Z., Elgamal, A. and Parra, E. (2003). Computational model for cyclic mobility and associated shear deformation. Journal of Geotechnical and Geoenvironmental Engineering 129(12), 1119-1127

Yen, W., Chen, G., Buckle, I., Allen, T., Alzamora, D., Ger, J. and Arias, J. (2011). Post-earthquake reconnaissance report on transportation infrastructure: impact of the February 27, 2010, Offshore Maule Earthquake in Chile. U.S Department of Transportation, Federal Highway Administration, USA

Youd, T. and Idris, I. (2001). Liquefaction resistance of soils: summary report from the 1996 NCEER and 1998 NCEER/NSF Workshops on Evaluation of Liquefaction Resistance of Soils. Journal of Geotechnical and Geoenvironmental Engineering 127(4), 297-313 